\title{
Delirium in older adults: a chronic cognitive disorder?
}

Delirium is defined as a neurocognitive disorder characterized by sudden onset, fluctuating course, and disturbances in level of consciousness, attention, orientation, memory, thought, perception, and behavior (American Psychiatric Association, 2013). It occurs in hyperactive, hypoactive, or mixed forms in up to $50 \%$ of older hospital patients (Inouye et al., 2014 ) and $70 \%$ of older long-term care residents (McCusker et al., 2011), many with pre-existing dementia (Fick et al., 2002).

The course of delirium is thought to be transient. Recovery is expected to be complete in the majority of affected individuals if the underlying putative cause (e.g. physical illness, drug toxicity) is promptly corrected or self-limited. The outcomes are presumed to be good (American Psychiatric Association, 2013). Among older adults, however, many well-conducted studies report that the outcomes of delirium are poor: delirium is associated with significant increases in cognitive impairment and functional disability, length of hospital stay, rates of institutionalization, and rates of death, independent of many socio-demographic and clinical variables (Witlox et al., 2010).

How to explain the poor outcomes? Increasing evidence suggests that delirium in a substantial proportion of older adults may be a chronic cognitive disorder and the persistence of delirium and symptoms of delirium may account for the poor outcomes.

\section{Persistent delirium}

Recovery from delirium refers to the sustained return to the pre-delirium level of mental function. Levkoff (Levkoff et al., 1992) and Rockwood (Rockwood, 1993) were among the first investigators to assess recovery systematically and reported that many older adults with delirium recovered in hospital but many others had not recovered by the time of discharge. More recently, a systematic review of 18 cohort studies reported that many older patients recovered from delirium but the combined proportions with persistent delirium at discharge from hospital and at 1,3 , and 6 months were $44.7 \%, 32.8 \%, 25.6 \%$, and $21 \%$, respectively (Cole et al., 2009). If the definition of persistent delirium includes patients with partial recovery, the frequencies of persistence may be even higher. In one study, the combined proportions with partial and no recovery from delirium at 1 and 3 months among patients with and without dementia were $85.9 \%$ and $72.7 \%$, and $67.9 \%$ and $51.9 \%$, respectively; predicted probabilities of partial and no recovery at 6 months were $62 \%$ and $37 \%$, respectively (Cole et al., 2015). Given the apparent asymptotic decrease in the frequencies of persistent delirium over time, it appears that a substantial proportion of older adults with delirium may never recover fully. The longer term outcomes (cognition, function, nursing home placement, mortality) of adults who recover appear to be good (Cole et al., $2008 \mathrm{~b}$ ) but the outcomes of adults who do not recover appear to be consistently worse than the outcomes of those who recover (Marcantonio et al., 2000; McCusker et al., 2003; McAvay et al., 2006).

\section{Persistent symptoms of delirium}

The emerging literatures on partial recovery from delirium, subsyndromal delirium (SSD) and persistent symptoms of delirium probably describe different facets of the same broad phenomenon: presence of symptoms of delirium (not meeting criteria for delirium), occurring either before or after episodes of delirium or independently of episodes of delirium. As for partial recovery, rates of partial recovery ranged from $20 \%$ to $42 \%$ at hospital discharge (Levkoff et al., 1992; O'Keeffe and Lavan, 1997; McAvay et al., 2006); rates of partial recovery at 12 and 24 weeks were $15 \%$ and $18 \%$, respectively (Cole et al., 2015). To date, there have been no studies of longer term outcomes but given the poor outcomes associated with persistent delirium, partial recovery is probably associated with poorer outcomes than full recovery.

As for SSD, estimates of prevalence in older adults range widely $(5 \%-61 \%)$, perhaps related to differences in diagnostic criteria or patient populations (Cole et al., 2013a; Zuliani et al., 2013; Meagher et al., 2014). In a systematic review, the combined estimate of prevalence was $23 \%$; the combined estimate of incidence was $13 \%$ (Cole et al., 2013a). SSD persisted at 12 weeks in $29 \%$ of older hospital inpatients (Cole et al., 2016) and at 24 weeks in $22 \%-25 \%$ of older long-term 
care residents (Cole et al., 2013b). Over time, many symptoms of SSD appear to become more frequent and persistent (Meagher et al., 2012; von Gunten et al., 2013). The longer term outcomes (cognition, function, length of hospital stay, rates of institutionalization, and death) of patients with SSD are poor and intermediate between the outcomes of patients with delirium versus no delirium (Cole et al., 2013a). Notably, the 6-month outcomes of patients who had not recovered from SSD at 8 weeks were worse than the outcomes of patients who had recovered (Cole et al., 2008a).

As to persistent symptoms, some symptoms of delirium persist after episodes of delirium in up to $65 \%$ of patients for at least 12 months, irrespective of the presence of dementia (Levkoff et al., 1992; Rockwood, 1993; McCusker et al., 2003; Cole et al., 2012a; Witlox et al., 2013). Moreover, symptoms often occur for long periods before episodes of delirium. One or more symptoms preceded $92.7 \%$ of incident episodes; the estimated median duration of symptoms before episodes was 27 weeks; the types of symptoms present were most often inattention or disorganized thinking (Cole et al., 2012b). The mean number of symptoms present appears to increase over time (Ciampi et al., 2011); the mean number of symptoms increases before episodes of delirium and is greater after episodes than before (Cole et al., 2012b). Persistent symptoms may occur independently of an identified episode of delirium: one or more symptoms were present in up to $67 \%$ of older adults without delirium and with or without dementia (Cole et al., 2002; Leonard et al., 2009; Meagher et al., 2014). Of note, the presence of one or more symptoms appears to be risk factor for incident delirium weeks or months later; the more symptoms present, the greater is the risk (Cole et al., 2013c). In the shorter term, persistent symptoms are associated with cognitive and functional impairment and mood and behavior problems, similar to full delirium or intermediate between full delirium and no symptoms (Cole et al., 2014). To date, there are no studies of the longer term outcomes but given the prognostic significance of even one symptom of delirium in the SSD literature, patients with persistent symptoms probably have poorer outcomes than patients with no symptoms.

Partial recovery, SSD, and persistent symptoms appear to have similar frequencies, courses, and outcomes in older adults. Often, the symptoms occur after episodes of delirium. If the symptoms persist for a long period of time, the temporal relationship between the symptoms and an episode of delirium may be lost; then, such symptoms may be seen as SSD, persistent symptoms, or symptoms that precede the next episode of delirium. Sometimes, the symptoms may occur independently of an episode of delirium, perhaps markers for underlying medical conditions (e.g. infection) not severe enough to cause full delirium; again, such symptoms may be seen as SSD or persistent symptoms. Over time, the symptoms appear to become more frequent and persistent. The presence of symptoms, whatever the label, appears to be associated with poorer outcomes.

\section{Proposed hypothesis}

Advances in knowledge depend on creative tension between observations and ideas (hypotheses). Observations cry out for explanation; a hypothesis is proposed; the hypothesis is tested by making more observations... and so on (Bernard, 1957). During the past 25 years, research on delirium in older adults has generated a mountain of observations. We propose that delirium in a substantial proportion (perhaps $20 \%-40 \%$ ) of many older adult populations with or without dementia is a chronic cognitive disorder characterized by a chronic fluctuating course, periods of acute exacerbation that are labeled episodes of delirium, increasing symptom frequency and persistence over time and poor outcomes. Figure 1 illustrates the course of this hypothesized condition. Some symptoms of delirium are present continuously, though fluctuating in number. At times, the number of symptoms present may cross the threshold for a diagnosis of delirium. Over time, the symptoms become more frequent and persistent.

Persistent symptoms of delirium may be markers for underlying medical conditions (e.g. hypoxia, drug toxicity) that may eventually become severe enough to cause full delirium. Alternatively, persistent symptoms may reflect abnormalities in neuroanatomical structures (Mesulam, 1985; MacLullich et al., 2008; Field et al., 2012) or neurotransmitter systems (Gaudreau and Gagnon, 2005; van Gool et al., 2010), or aberrant stress responses (i.e. exaggerated brain inflammatory responses, LPHA axis dysfunction, MacLullich et al., 2008) that may interact with putative precipitating conditions (e.g. urinary tract infection) to induce delirium. Aberrant stress responses are potentially neurotoxic (Barrientos et al., 2006; MacLullich et al., 2008). Persistent delirium, partial recovery, SSD, and persistent symptoms may reflect the persistence of neurotoxicity. Persistence of neurotoxicity may lead to brain atrophy and white matter disruption (Morandi et al., 2012) and poorer outcomes.

The putative precipitating conditions above may be classified into two categories (MacLullich et al., 


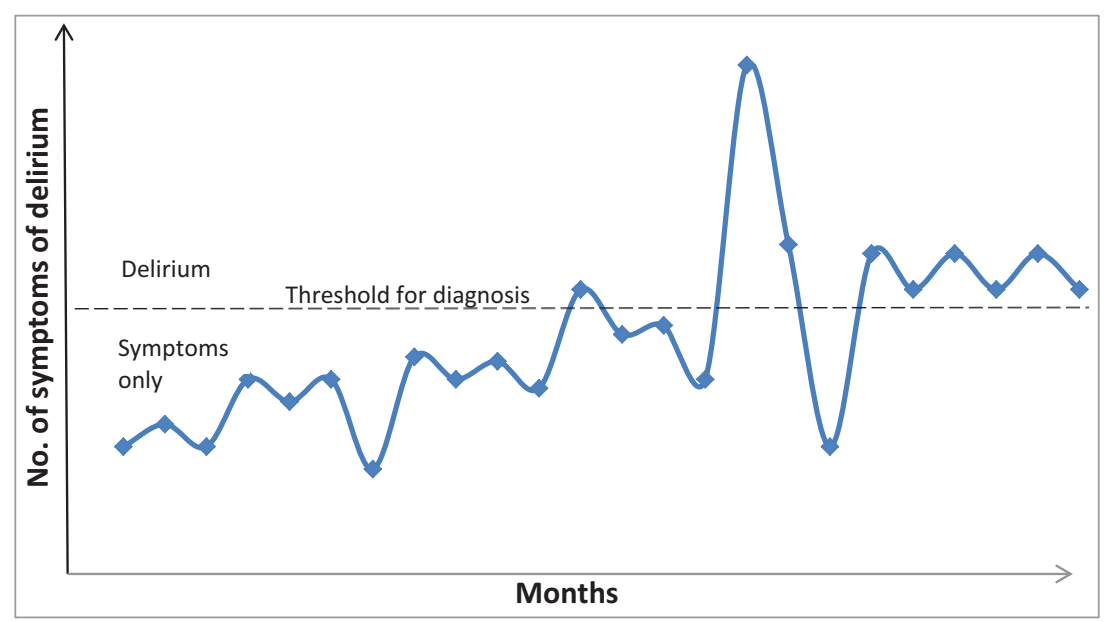

Figure 1. (Colour online) Hypothetical course of persistent symptoms of delirium in one older adult.

2008): direct neuronal insults (e.g. hypoxia, trauma, drug toxicity) that induce delirium directly and systemic illnesses (e.g. infection, organ failure) that induce delirium by interacting with abnormalities in neuroanatomical structures, neurotransmitter systems, or stress responses. Delirium induced by direct neuronal insults may be more likely to recover fully if the putative cause is promptly treated or self-limited whereas delirium induced by systemic illnesses may be more persistent. A recent study attempted to explore the relationship between putative causes of delirium and recovery but small sample size prevented definitive conclusions; indeed, the only putative cause significantly associated with partial or no recovery in one model was fluid/electrolyte abnormality, a finding seemingly inconsistent with the above proposal (Cole et al., 2015).

Interestingly, the proposed hypothesis may be analogous to current understanding of the pathogenesis and course of chronic obstructive pulmonary disease (COPD). COPD is characterized by a chronic fluctuating course with periods of acute exacerbation. Exacerbations result from exposure to noxious agents (e.g. bacteria, air pollution), increases in inflammatory mediators, airway wall infiltration, and decreased airflow. Repeated exacerbations may accelerate decline in lung function (Papi et al., 2006).

\section{Clinical implications}

The proposed hypothesis has implications for clinical practice. First, it suggests that current approaches to prevention and treatment of delirium in a large proportion of older adults may address only factors leading to exacerbation of symptoms beyond the threshold for diagnosis. Second, it is possible that persistent symptoms and the associated cognitive and functional impairment may interfere with patient self-management of chronic medical conditions (e.g. poor compliance with diet or medication) which may, in turn, contribute to poor outcomes. Third, it may be useful to explore the application of elements of the chronic disease management model to the care of older patients who have persistent symptoms of delirium such as new clinical information systems to monitor delirium symptoms, decision support algorithms for patients who continue to have symptoms, and delivery system redesign to support interventions (Bodenheimer et al., 2002). On this point, a small quasi-experimental trial provides preliminary evidence of the potential benefit of continuing care of older adults who have had an episode of delirium in terms of delayed institutionalization (Rahkonen et al., 2001). Finally, patients at risk of persistent delirium and delirium symptoms may become the focus of preventive interventions modeled on interventions to prevent delirium (Inouye et al., 1999).

\section{Research implications}

The proposed hypothesis has implications for research. First, delirium may not be a unitary condition: persistent symptoms may identify a delirium sub-type with an unique etiology and pathogenesis, and poor prognosis; controvertibly, persistent symptoms may be a marker for an underlying brain vulnerability such as dementia. Second, it may be important to monitor the longer term course (e.g. 24-36 months) of delirium symptoms to document any increases in frequency 
and persistence; however, better methods (e.g. type and frequency of follow-up assessments) and measures (e.g. a delirium instrument designed to measure symptom persistence) will be required to study the presence and course of persistent symptoms. Third, it is not clear whether the persistence of symptoms is related to chronic medical conditions (e.g. infection, drug toxicity) or abnormalities in neuroanatomical structures, neurotransmitter systems, or stress responses. It may be useful to explore whether interventions to manage chronic medical conditions or moderate the predisposing abnormalities can increase rates of full recovery and improve longer term outcomes. Fourth, there may be a dose-response relationship between the severity and duration of symptoms, recovery status, and longer term outcomes. Greater severity of symptoms has been associated with partial and no recovery (Cole et al., 2015); greater severity and duration of symptoms has been associated with poorer long-term outcomes (McCusker et al., 2003; Morandi et al., 2012); SSD, a clinical state characterized by the presence of symptoms not meeting criteria for delirium, has outcomes intermediate between full delirium and no symptoms (Cole et al., 2013a). Thus, development of pharmacologic and nonpharmacologic strategies to decrease the severity and duration of symptoms of delirium may lead to better longer term outcomes (Friedman et al., 2014).

\section{Conclusion}

Is delirium a chronic cognitive disorder? Current evidence suggests that many older adults with delirium may recover fully and have good outcomes but in a substantial proportion of older adults delirium may be a chronic cognitive disorder characterized by a chronic fluctuating course, periods of acute exacerbation, increasing symptom frequency, and persistence over time and poor outcomes.

\section{Conflict of interest}

None.

\author{
MARTIN G. COLE ${ }^{1}$ AND JANE MCCUSKER ${ }^{2}$ \\ ${ }^{1}$ Department of Psychiatry, St Mary's Hospital and \\ McGill University, Montreal, Canada \\ Email: martin.cole@ssss.gouv.qc.ca \\ ${ }^{2}$ St. Mary's Research Center, Department of \\ Epidemiology and Biostatistics, McGill University, \\ Montreal, Canada \\ Email: jane.mccusker@mcgill.ca
}

\section{References}

American Psychiatric Association (2013). Diagnostic and Statistical Manual of Mental Disorders (DSM-V). Washington, DC: American Psychiatric Press.

Barrientos, R. M. et al. (2006). Peripheral infection and aging interact to impair hippocampal memory consolidation. Neurobiology of Aging, 27, 723-732.

Bernard, C. (1957). An Introduction to the Study of Experimental Medicine. New York: Dover Publications.

Bodenheimer, T., Wagner, E. H. and Grumbach, K. (2002). Improving primary care for patients with chronic illness. FAMA, 288, 1775-1779.

Ciampi, A., Dyachenko, A., Cole, M. and McCusker, J. (2011). Delirium superimposed on dementia: defining disease states and course from longitudinal measurements of a multivariate index using latent class analysis and hidden Markov chains. International Psychogeriatrics, 23, 1659-1670.

Cole, M. G. et al. (2015). Partial and no recovery from delirium in older hospitalized adults: frequency and baseline risk factors. Fournal of the American Geriatrics Society, 63, 2340-2348.

Cole, M. G. et al. (2016). Frequency of full, partial and no recovery from subsyndromal deliriumin older hospital inpatients. International fournal of Geriatric Psychiatry, 31, 544-550.

Cole, M. G., Ciampi, A., Belzile, E. and Dubuc-Sarrasin, M. (2013a). Subsyndromal delirium in older people: a systematic review of frequency, risk factors, course and outcomes. International fournal of Geriatric Psychiatry, 28, 771-780.

Cole, M. G., Ciampi, A., Belzile, E. and Zhong, L. (2009). Persistent delirium in older hospital patients: a systematic review of frequency and prognosis. Age and Ageing, 38, 19-26.

Cole, M. G., McCusker, J., Ciampi, A. and Belzile, E. (2008a). The 6- and 12-month outcomes of older medical inpatients who recover from subsyndromal delirium. Fournal of the American Geriatrics Society, 56, 2093-2099.

Cole, M. G., McCusker, J., Dendukuri, N. and Han, L. (2002). Symptoms of delirium among elderly medical inpatients with or without dementia. Fournal of Neuropsychiatry and Clinical Neurosciences, 14, 167-175.

Cole, M. G. et al. (2012a). The course of delirium in older long-term care residents. International fournal of Geriatric Psychiatry, 27, 1291-1297.

Cole, M. G. et al. (2012b). Symptoms of delirium occurring before and after episodes of delirium in older long-term care residents. Fournal of the American Geriatrics Society, 60, 2302-2307.

Cole, M. G. et al. (2013b). The course of subsyndromal delirium in older long-term care residents. The American Fournal of Geriatric Psychiatry, 21, 289-296.

Cole, M. G. et al. (2013c). Symptoms of delirium predict incident delirium in older long-term care residents. International Psychogeriatrics, 25, 887-894.

Cole, M. G. et al. (2014). Core symptoms not meeting criteria for delirium are associated with cognitive and functional impairment and mood and behavior problems in older long-term care residents. International Psychogeriatrics, $26,1181-1189$. 
Cole, M. G., You, Y., McCusker, J., Ciampi, A. and Belzile, E. (2008b). The 6 and 12 month outcomes of older medical inpatients who recover from delirium. International fournal of Geriatric Psychiatry, 23, 301-307.

Fick, D. M., Agostini, J. V. and Inouye, S. K. (2002). Delirium superimposed on dementia: a systematic review. Fournal of the American Geriatrics Society, 50, 1723-1732.

Field, R. H., Gossen, A. and Cunningham, C. (2012). Prior pathology in the basal forebrain cholinergic system predisposes to inflammation-induced working memory deficits: reconciling inflammatory and cholinergic hypotheses of delirium. Fournal Neurosci, 32, 6288-6294.

Friedman, J. I., Soleimani, L., McGonigle, D. P., Egol, C. and Silverstein, J. H. (2014). Pharmacological treatments of non-substance-withdrawal delirium: a systematic review of prospective trials. American fournal of Psychiatry, 171, 151-159.

Gaudreau, J. D. and Gagnon, P. (2005). Psychotogenic drugs and delirium pathogenesis: the central role of the thalamus. Medical Hypotheses, 64, 471-475.

Inouye, S. K. et al. (1999). A multicomponent intervention to prevent delirium in hospitalized older patients. New England Fournal of Medicine, 340, 669-720.

Inouye, S. K., Westendorp, R. G. and Saczynski, J. S. (2014). Delirium in elderly people. Lancet, 383, 911-922.

Leonard, M., Spiller, J., Keen, J., MacLullich, A., Kamholtz, B. and Meagher, D. (2009). Symptoms of depression and delirium assessed serially in palliative-care inpatients. Psychosomatics, 50, 506-514.

Levkoff, S. E. et al. (1992). Delirium: the occurrence and persistence of symptoms among elderly hospitalized patients. Archives of Internal Medicines, 152, 334-340.

MacLullich, A. M., Ferguson, K. J., Miller, T., de Rooij, S. E. and Cunningham, C. (2008). Unravelling the pathophysiology of delirium: a focus on the role of aberrant stress responses. Fournal of Psychosomatic Research, 65, 229-238.

Marcantonio, E. R., Flacker, J. M., Michaels, M. and Resnick, N. M. (2000). Delirium is independently associated with poor functional recovery after hip fracture. Fournal of the American Geriatrics Society, 48, 618-624.

McAvay, G. J. et al. (2006). Older adults discharged from the hospital with delirium: 1-year outcomes. Fournal of the American Geriatrics Society, 54, 1245-1250.

McCusker, J., Cole, M. G., Dendukuri, N., Han, L. and Belzile, E. (2003). The course of delirium in older medical inpatients: a prospective study. Fournal of General Internal Medicine, 18, 696-704.

McCusker, J. et al. (2011). Prevalence and incidence of delirium in long-term care. International fournal of Geriatric Psychiatry, 26, 1152-1161.
Meagher, D., Adamis, D., Trzepacz, P. and Leonard, M. (2012). Features of subsyndromal and persistent delirium. The British Fournal of Psychiatry, 200, 37-44.

Meagher, D. et al. (2014). Frequency of delirium and subsyndromal delirium in an adult acute hospital population. The British fournal of Psychiatry, 205, 478-485.

Mesulam, M. M. (1985). Attention, confusional states, and neglect. In Principles of Behavioral Neurology (Chapter 3, pp. 125-168). Philadelphia: FA Davis Co.

Morandi, A. et al. (2012). The relationship between delirium duration, white matter integrity, and cognitive impairment in intensive care unit survivors as determined by diffusion tensor imaging: the VISIONS prospective cohort magnetic resonance imaging study. Critical Care Medicine, 40, 2182-2189.

O'Keeffe, S. and Lavan, J. (1997). The prognostic significance of delirium in older hospital patients. Fournal of the American Geriatrics Society, 45, 174-178.

Papi, A., Luppi, F., Franco, F. and Fabbri, L. M. (2006). Pathophysiology of exacerbations of chronic obstructive pulmonary disease. Proceedings of the American Thoracic Society, 3, 245-251.

Rahkonen, T., Elonienmi-Sulkaya, U., Paanila, S., Halonen, P., Sivenius, J. and Sulkava, R. (2001). Systematic intervention for supporting community care of elderly people after a delirium episode. International Psychogeriatrics, 13, 37-49.

Rockwood, K. (1993). The occurrence and duration of symptoms in elderly patients with delirium. Fournal of Gerontology, 48, M162-M166.

van Gool, W. A., van de Beek, D. and Eikelenboom, P. (2010). Systemic infection and delirium: when cytokines and acetylcholine collide. Lancet, 375, 773-775.

von Gunten, A., Mosimann, U. P. and Antionietti, J. (2013). A longitudinal study on delirium in nursing homes. American fournal of Geriatric Psychiatry, 21, 963-972.

Witlox, J., Eurelings, L. S., de Jonghe, J. F., Kalisvaart, K. J., Eikelenboom, P. and van Gool, W. A. (2010). Delirium in elderly patients and the risk of postdischarge mortality, institutionalization, and dementia: a meta-analysis. FAMA, 304, 443-451.

Witlox, J. et al. (2013). The neuropsychological sequelae of delirium in elderly patients with hip fracture three months after hospital discharge. International Psychogeriatrics, 25, 1521-1531.

Zuliani, G. et al. (2013). Subsyndromal delirium and its determinants in elderly patients hospitalized for acute medical illness. The fournals of Gerontology. Series A, Biological Sciences and Medical Sciences, 68, 12961302. 